ORIGINAL

\title{
Prevalência das afecções podais em vacas leiteiras da região sul do estado do Espírito Santo, Brasil
}

\author{
Prevalence of foot diseases in dairy cattle in the southern region of Espírito \\ Santo state, Brazil
}

\author{
Pricilla Nascimento Pozzatti ${ }^{1}$, Fernando Passon Casagrande², Marcus Dantas Dórea Correio ${ }^{3}$, Luiz Felipe Nunes \\ Menezes Borges ${ }^{4}$, Lenir Cardoso Porfírio ${ }^{2}$, Hyran Bernardes Pinheiro ${ }^{5}$ Paulo Cesar Amaral Ribeiro da Silva ${ }^{5^{*}}$ \\ ${ }^{1}$ Instituto Federal Catarinense (IFC), Araquari, SC, Brasil \\ 2 Universidade Federal do Espírito Santo (UFES), Vitória, ES, Brasil \\ ${ }^{3}$ Dórea Veterinária Ltda, Teixeira de Freitas, BA, Brasil \\ ${ }^{4}$ Secretaria Municipal de Saúde, Juatuba, MG, Brasil \\ ${ }^{5}$ Universidade Federal Fluminense (UFF), Niterói, RJ, Brasil
}

\section{Resumo}

O presente estudo teve como objetivo principal realizar o levantamento das principais afecções podais que acometem os bovinos na região do sul capixaba do estado do Espírito Santo. Foram avaliadas propriedades leiteiras contemplando diferentes sistemas de produção, classificados nas categorias extensivo, semiconfinamento e confinamento. De 1225 vacas leiteiras examinadas, 25 apresentaram pelo menos um tipo de lesão podal, identificando-se um total de 64 lesões. A prevalência das afecções podais foi de 2,04\%. Das afecções de casco, 82,10\% ocorreram nos membros posteriores e $17,90 \%$ nos membros anteriores. As afecções de maior frequência encontradas no presente estudo foram erosão de talão (18,75\%), dermatite interdigital (14,07\%), pododermatite séptica e dermatite digital (10,94\% cada), e úlcera de sola $(9,37 \%)$. O conhecimento da prevalência das afecções podais na região sul do Espírito Santo nos permite identificar possíveis falhas de manejo existentes e estabelecer medidas profiláticas de maneira mais eficiente.

Palavras-chave: Epidemiologia. Doenças de casco. Gado leiteiro.

\section{Abstract}

The main objective of this study was to carry out a survey of the main foot conditions affecting cattle in the southern region of the Espírito Santo state, Brazil. Dairy properties were evaluated considering different production systems classified as extensive, semi-confinement, and confinement. 
Of 1225 dairy cows examined, 25 had at least one type of foot disorder, and a total of 64 lesions were identified. The prevalence of foot conditions was $2.04 \% .82 .10 \%$ of the hull affections occurred in the hind limbs and $17.90 \%$ in the anterior limbs. The most frequent affections found in the present study were bead erosion (18.75\%), interdigital dermatitis (14.07\%), septic pododermatitis and digital dermatitis (10.94\% each), and sole ulcer (9.37\%). Knowing the prevalence of foot conditions in the southern region of Espírito Santo state allows us to identify possible management flaws and establish prophylactic measures more efficiently.

Keywords: Epidemiology. Claw diseases. Dairy cattle.

\section{Introdução}

A abertura da economia para o mercado internacional aumentou a concorrência e passou a exigir maior produtividade com melhor qualidade nos diferentes segmentos produtivos. $\mathrm{Na}$ bovinocultura leiteira, com o propósito de alcançar tais avanços, os criadores vem implementando métodos de criação ditos modernos, mas muitas vezes utilizados de forma inadequada, influenciando diretamente as condições epidemiológicas favoráveis à ocorrência de várias enfermidades podais e resultando em grandes prejuízos para os criatórios (Silva et al., 2001).

Embora considerado como problema que afete o comportamento e o desempenho dos animais, as desordens digitais dos bovinos são negligenciadas e subestimadas. Esta tendência é provavelmente devido às dificuldades com a manipulação dos cascos dos bovinos (Bergsten et al., 1998). Além de consideradas um dos maiores problemas de saúde em gado leiteiro (Faye e Lescourret, 1989), as lesões no casco são responsáveis por aproximadamente $90 \%$ das claudicações nos bovinos, sendo os prejuízos econômicos traduzidos por queda na produção, custo do tratamento (serviços profissionais e medicamentos), leite descartado por resíduos de antibióticos, perda de escore corporal, problemas reprodutivos (como ausência de cio), maior número de serviços por prenhez e maior intervalo entre partos nas fêmeas, pior qualidade do sêmen nos machor, descarte precoce do animal, custo de reposição e maior susceptibilidade a outras doenças (Greenough et al., 1983; Nicoletti, 2004). Conforme a Organização das Nações Unidas para a Alimentação e a Agricultura (FAO, 1967), o total de perdas provocadas pelas afecções podais em vacas atinge $15 \%$ da produção em países desenvolvidos e 30 a $40 \%$ nos países em desenvolvimento. 0 problema ocorre com alta prevalência no Brasil (Silveira et al., 1988), tendo maior frequência em bovinos mantidos em sistemas de criação confinados e semiconfinados (Mgassa et al., 1988; Ribeiro et al., 1992).

O confinamento e a predominância de animais de raças europeias no rebanho são os fatores mais importantes relacionados com o aumento de afecções do aparelho locomotor em vacas de leite (Arkins,1981; Rowlands et al., 1983; Silveira et al., 1988). Bovinos criados em confinamento que conseguem manter um balanço adequado entre crescimento e desgaste do estojo córneo são menos sujeitos a problemas de cascos. As más condições de higiene e superlotação dos currais favorecem a instalação de infecções podais específicas, como flegmão interdigital, dermatite interdigital e dermatite digital papilomatosa (Nicoletti, 2004). Em sistemas de criação intensiva de bovinos, em especial de raças leiteiras, o corte de casco preventivo é salutar e necessário para diminuir a frequência de problemas locomotores, assim como o descarte de animais por esses motivos (Greenough e Weaver, 1997).

Segundo Greenough (2007), no período de transição, que compreende as últimas quatro semanas de gestação e as quatro primeiras de lactação, as papilas rumenais não estão alongadas o suficiente. Isto leva a uma menor absorção dos ácidos graxos voláteis e consequente dimiminuição do $\mathrm{pH}$ rumenal, caracterizando uma acidose que pode vir a favorecer a liberação de endotoxinas. A endotoxemia terá ação direta na microcirculação do casco junto a outras substâncias inflamatórias e, então, conforme o aumento da pressão intraungular, ocorre diminuição do fluxo capilar. Com a vascularização afetada e dilatada ao máximo, há o extravasamento de sangue e fluído com formação de trombos, podendo levar à isquemia e necrose. 
Allenstein (1981), Edwards (1982), Liversey e Fleming (1984) também apontaram que a histamina e endotoxinas, que são substâncias vasoativas e produzidas em decorrência das doenças sistêmicas no período pós-parto, tais como acetonemia, mastite, metrite e acidose rumenal, são responsáveis por danos ao cório laminar. Os autores chamaram atenção para o fato da laminite aguda ter sido observada em animais portadores de doenças em que essa modalidade de endotoxina podia estar presente. Vermunt e Greenough (1994) verificaram a ocorrência de laminites em bovinos submetidos a altas doses de endotoxinas administradas via artérias digitais.

Os membros que possuem maior acometimento em gado leiteiro são os pélvicos, o que, para Borges (1998), deve-se ao maior contato destes com fezes, urina e excesso de umidade, principalmente durante a ordenha. Baggott e Russell (1981) complementam esses dados observando que em animais desta categoria de produção as lesões localizadas nos cascos laterais são 2,5 vezes mais frequentes em relação às encontradas nos cascos mediais. Lima et al. (2009) salientam que estudos radiográficos auxiliam no diagnóstico mais preciso das claudicações.

Estudos que correlacionam genética e afecções podais demonstram que apesar da predisposição genética a lesões de casco, os valores de herdabilidade para tal são baixos, indicando que o fator ambiental e manejo são mais importantes no aparecimento das lesões. De acordo com Oberbauer et al. (2013), o valor encontrado de herdabilidade $\left(\mathrm{h}^{2}\right)$ é de 0,03 a 0,4 conforme o tipo de lesão. Tais valores também ratificam os estudos feitos por Buch et al. (2011), que encontraram valores para todas as lesões de casco variando entre 0,03 a 0,05 . Schöpke et al. (2013) obtiveram valores próximos para $h^{2}$, variando entre 0,06 e 0,14 .

A escassez de estudos que descrevam a prevalência das afecções podais em bovinos no estado do Espírito Santo ressalta a necessidade e importância epidemiológica deste trabalho. 0 objetivo deste estudo foi estabelecer a prevalência das afecções podais em bovinos leiteiros na região sul capixaba. Atualmente, são escassos os trabalhos que relatam estas afecções na região, o que é fundamental para orientar as atitudes a serem estabelecidas na prevenção e controle.

\section{Materiais e métodos}

Foram avaliadas propriedades leiteiras sob o sistema de produção extensivo, semiconfinamento e em confinamento da região sul do Espírito Santo, com o objetivo de realizar o levantamento das principais afecções podais em bovinos nessa região. Foram examinadas 1225 vacas Girolando com variados graus de sangue, oriundas de 18 propriedades leiteiras das cidades de Alegre, Irupi, Divino de São Lourenço, Guaçuí, Ibitirama e Iuna, na região sul capixaba, onde foram verificadas as condições de manejo, alimentação e sanidade dos animais. Posteriormente, os animais foram examinados individualmente, sendo feita a limpeza dos cascos acometidos, e as lesões foram classificadas segundo os critérios de Greenough et al. (1983): 1. Dermatite interdigital - inflamação da pele interdigital sem extensão aos tecidos profundos; 2. Erosão de camada córnea - perda de substância da camada córnea da sola ou dos talões; 3. Dermatite verrucosa - inflamação crônica da pele na região dorsal ou palmar/plantar; 4. Hiperplasia interdigital - reação proliferativa da pele e/ou do tecido subcutâneo interdigital; 5. Dermatite digital - inflamação circunscrita ou difusa da coroa do casco; 6. Flegmão interdigital - inflamação da pele interdigital e dos tecidos subjacentes, caracterizada por necrose de pele com fissura; 7. Pododermatite asséptica difusa inflamação asséptica aguda, subaguda ou crônica da pododerme; 8. Pododermatite circunscrita - ulceração circunscrita da pododerme; 9. Pododermatite séptica - inflamação séptica, difusa ou localizada da pododerme; 10. Fissura da unha - fissura da camada córnea da muralha paralela à sua face dorsal ou paralela à coroa; 11. deformação da unha - qualquer tipo de deformação da unha; 12. Afecções diversas - outras afecções que não se encontram descritas acima.

Os dados individuais foram agrupados e analisados através de porcentagem. 


\section{Resultados e discussão}

No presente trabalho, a prevalência de animais com lesões podais foi de 2,04\%. Das afecções de casco, $82,10 \%$ ocorreram nos membros posteriores e $17,90 \%$ nos membros anteriores. Das 18 propriedades avaliadas, 33,33\% tinham sistema de manejo extensivo, 55,55\% semi-intensivo e $11,12 \%$ intensivo, tendo $16,67 \%, 40 \%$ e $100 \%$ de problemas podais, respectivamente, em cada modelo de criação. Dos 1225 animais avaliados, 25 apresentaram pelo menos um tipo de lesão podal, identificando-se 64 lesões (Tabela 1).

Tabela 1 - Ocorrência e porcentagens de lesões podais em vacas na região sul capixaba do Espírito Santo

\begin{tabular}{lcc}
\hline Lesões & Casos & $\%$ \\
\hline Erosão de talão & 12 & 18,75 \\
Dermatite interdigital & 09 & 14,07 \\
Pododermatite séptica & 07 & 10,94 \\
Dermatite digital & 07 & 10,94 \\
Úlcera de sola & 06 & 9,37 \\
Hiperplasia interdigital & 05 & 7,81 \\
Dupla sola & 04 & 6,25 \\
Doença da linha branca & 04 & 6,25 \\
Úlcera de talão & 03 & 4,69 \\
Úlcera de pinça & 02 & 3,12 \\
Flegmão interdigital & 02 & 3,12 \\
Anormalidade de casco & 02 & 3,12 \\
Pododermatite do paradígito & 01 & 1,57 \\
\hline Total & 64 & 100 \\
\hline
\end{tabular}

Todas as propriedades apresentavam higienização deficiente das instalações e dos piquetes. A realização do exame clínico individual e específico foi decisiva para a classificação das lesões e para o estabelecimento do diagnóstico.

A prevalência observada neste estudo de 2,04\% dos animais com problemas podais foi bem abaixo da encontrada por Silva et al. (2001) no estado de Goiás, que foi de 29,67\%. Entretanto, Machado et al. (2008) encontraram prevalência de 5,08\% em 1240 fêmeas na região da bacia leiteira do município de Itapecuru Mirim, no Maranhão. Dias (1996) descreveu que as doenças dos cascos acometeram de $11 \%$ a $25 \%$ das vacas de todas as propriedades de seu estudo, mas não fez menção sobre a aptidão e o manejo do rebanho. Já Confalonieri et al. (2016), em estudo realizado com 12132 animais em confinamento, encontraram uma pravelência de $2,71 \%$ em gado leiteiro. Por outro lado, Molina et al. (1999), utilizando 469 vacas da raça Holandesa de dez propriedades rurais, todas em lactação e em confinamento, encontraram uma prevalência de $30,28 \%$. Em estudo feito na bacia leiteira em Rondon, Pará, com 1236 vacas zebuínas mestiças, com predominância da raça Indubrasil, Silveira et al. (2009) encontraram prevalência de lesões podais em $22,5 \%$ dos animais. As prevalentes divergências nos trabalhos se justificam pelos diferentes sistemas de manejo e raças dos rebanhos estudados, o que pode ser ratificado por Arkins (1981), Rowlands et al. (1983) e Silveira et al. (1988), que afirmam que o confinamento e a predominância de animais de raças europeias no rebanho são os fatores mais importantes relacionados ao aumento de afecções do aparelho locomotor em vacas de leite.

De acordo com Mauchle et al. (2008), além dos fatores ambientais, os fatores genéticos e nutricionais são siginifcativos para o desenvolvimento das lesões. Vários fatores como anatomia, genética, idade, peso corporal e características ambientais exercem influência sobre a conformação do casco (Casagrande, 2010). De acordo com O'Driscoll et al. (2010), outro fator de manejo que chamou atenção para o aparecimento de lesões de casco foi que vacas ordenhadas uma vez ao dia apresentavam uma melhor saúde dos cascos e dos quadris.

No presente estudo, identificou-se um maior número de lesões nos membros pélvicos $(82,10 \%)$ do que nos torácicos (17,90\%), valores bem próximos aos achados por Marega (2001), que encontrou 87,66\% dos membros pélvicos acometidos. Da mesma forma, Molina et al. (1999), Rabelo et al. (2013) e Silveira et al. (2009) identificaram mais problemas nos cascos dos membros pélvicos $(66,67 \%, 75,3 \%, 61,83 \%$, respectivamente) do que nos membros torácicos $(33,33 \%, 24,7 \%, 38,17 \%$, respectivamente). Tais valores podem ser explicados pela probabilidade de o maior acometimento dos membros pélvicos ocorrer pelo maior contato com fezes, urina e excesso de umidade (Borges, 1998). 
As afecções de maior ocorrência foram erosão de talão, com $18,75 \%$ do total, seguida da dermatite interdigital (14,07\%) e da pododermatite séptica e dermatite digital, ambas com 10,94 \%. Molina et al. (1999) também encontraram como lesão mais frequente a erosão da camada córnea, seguida por dermatite interdigital e pododermatite séptica. Jubb e Malmo (1991) examinaram 653 vacas produtoras de leite, alimentadas a pasto, e identificaram a dermatite digital, dermatite e hiperplasia interdigital como as lesões mais frequentes. Silva et al. (2001), por sua vez, encontraram com mais frequência a dermatite digital, seguida da dermatite verrucosa e da pododermatite séptica.

Já Albuquerque et al. (2009), ao avaliarem 66 animais da raça Holandesa em sistema de confinamento, observaram que a doença de maior ocorrência foi a doença da linha branca, seguida de erosão de talão e úlcera de sola. Em um estudo retrospectivo com 209 casos de afecções locomotoras, Gargano et al. (2013) encontraram maior prevalência para hiperplasia interdigital, úlcera de sola e dermatite interdigital. Greenough (1987), Corbellini (1994) e Moraes (2000) acreditam que bactérias como Fusobacterium necrophorum e Dichelobacter nodosus também estejam envolvidas na etiologia das enfermidades podais. Entretanto, Alves et al. (2007) identificaram alta frequência de Prevotella melaninogênica, Porphyromonas sp. e Proteus sp. em pododermatites em vacas leiteiras.

Neste estudo, identificou-se que em 100\% das propriedades com sistema de produção intensivo os animais apresentavam lesões podais. De acordo com Shearer e Amstel (2000), ambientes com piso de concreto contribuem para o desgaste dos cascos e propiciam o aparecimento de úlceras de sola e doenças da linha branca. Além disso, segundo Allenstein (1981), outro desafio é a limpeza dos confinamentos. Com a utlização de água, há o problema da umidade excessiva, que contribui para o amolecimento dos cascos. Em contrapartida, a remoção de dejetos realizada somente por raspagem também pode favorecer a ocorrência de problemas de casco, visto não ser normalmente suficiente para evitar o acúmulo de grande quantidade de matéria orgânica no piso das instalações.

\section{Conclusão}

O conhecimento da prevalência de afecções podais no estado do Espírito Santo é indispensável para o desenvolvimento do tratamento e prevenção de doenças, visto que as enfermidades de cascos acarretam maior custo com o tratamento dos animais doentes, diminuição na produção leiteira e redução na taxa de concepção. No presente estudo, a baixa prevalência de afecções podais ocorreu pelo reduzido número de propriedades em sistema de confinamento.

\section{Referências}

Albuquerque PI, Ximenes FHB, Moscardini ACR, Gouvêa LV, Mota ALAA, Godoy RF, et al. Caracterização das afecções podais em rebanho de gado holandês confinado. Cienc Anim Bras. 2009;(Supl 1):46-52.

Allenstein LC. Lameness of cattle. Can Vet J. 1981;22(3):65-7.

Alves CGT, Silva FF, Melo LEH, Santos NVM, Silva Jr FF, Machado PP, et al. Identificação de bactérias em afecções podais de vacas leiteiras. Med VetUFRPE.2007;1(2):16-20.

Arkins S. Lameness in dairy cows. Ir Vet J. 1981;35:163-170.

Baggott DG, Russell AM. Lameness in Cattle. Br Vet J. 1981;137(1):113-32.

Bergsten C, Hancock DD, Gay JM, Gay CC, Fox L. Claw diseases: the most common cause of dairy lameness diagnoses, frequencies and risk groups in a University herd. Proc Bov Pract. 1988;31:188-94.

Borges NC. Caracterização do fluido rumenal e dos parâmetros clínicos-laboratoriais de bovinos com pododermatite [dissertação]. Goiânia: Universidade Federal de Goiás; 1998. 69 p.

Buch LH, Sørensen AC, Lassen J, Berg P, Eriksson JÅ, Jakobsen JH, et al. Hygiene-related and feed-related hoof diseases show different patterns of genetic correlations to clinical mastitis and female fertility. J Dairy Sci. 2011;94(3):1540-51. 
Casagrande FP. Conformação dos cascos e das pernas de fêmeas bovinas holandesas em diferentes idades e prevalência de lesões [dissertação]. Belo Horizonte: Universidade Federal de Minas Gerias; 2010. 95 p.

Confalonieri OE, Moscuzza H, Rodríguez EM, Passucci JA. Patologías podales en ganado lechero y feedlot del partido de Tandil, provincia de Buenos Aires, Argentina. Rev Med Vet Zoot. 2016;63(1):11-9.

Corbellini CN. Factores nutricionales de riesgo para las afecciones podales. In: Enfermidades podales del bovino. Jornada-Taller para médicos veterinários; Navarro; 1994. p. 1-15.

Dias RS. Tratamento de cascos se faz com informações e critérios. Revta Balde Branco. 1996;385(3):26-2.

Edwards GB. Acute and subacute laminitis in cattle. Vet Ann. 1982;22:99-106.

FAO. Animal health yearbook. Roma: FAO; 1967. 332 p.

Faye B, Lescourret F. Environmental factors associated with lameness in dairy cattle. Prev Vet Med. 1989;7(4):267-87.

Gargano RG, Benesi FJ, Birgel Junior EH, Libera AMMPD, Gregory L, Sucupira MCA, et al. Estudo retrospectivo das afecções locomotoras em ruminantes atendidos na Faculdade de Medicina Veterinária e Zootecnia da Universidade de São Paulo entre 2000 e 2012. Braz J Vet Res Anim Sci. 2013;50(4):286-93.

Greenough PR. Bovine laminitis and lameness - A handson approach. Philadelphia: Saunders; 2007. 328 p.

Greenough PR. Pododermatitis circmscripta (Ulceration of the sole) in cattle. Agri-Practice; 1987;17-22.

Greenough PR, MacCallum FJ, Weaver AD. Les boiteries des bovins. Paris: Éditions Du Point Veterinaire; 1983. 478 p.

Greenough PR, Weaver AD. Lameness in cattle. Philadelphia: W.B. Saunders; 1997. 336 p.

Jubb TE, Malmo J. Lesions causing lameness requiring veterinary treatment in pasture-fed dairy cows in East Gippsland. Aust Vet J. 1991;68(1):21-4.
Lima IR, Fonseca AM, Silva LH, Costa APA, Silva LAF, Borges NC. Estudo radiográfico das extremidades distais dos membros locomotores de bovinos com claudicação. Cienc Anim Bras. 2009;(Supl 1):423-9.

Liversey CT, Fleming FL. Nutritional influences on laminitis, sole ulcer and bruised sale in Friesian cows. Vet Rec. 1984;114(21):510-2.

Machado PP, Pereira HM, Santos HP, Oliveira RA, Guerra PC, Teixeira WC. Prevalência e classificação de afecções podais em fêmeas bovinas destinadas à produção de leite na bacia leiteira do município de Itapecuru Mirim-MA. Rev Bras Saude Prod An. 2008;9(4): 777-86.

Marega LM. Aspectos epidemiológicos e de tratamento da dermatite digital em bovinos [dissertação]. Jaboticabal: Universidade Estadual Paulista; 2001. 68 p.

Mauchle U, Carvalho AU, Alzamora Filho F, Ferreira PM, Facury Filho EJ, Cavalcante MP. Efeito da sazonalidade sobre a ocorrência de lesões podais em vacas de raças leiteiras. Rev Bras SaUde Prod An. 2008;9(1):109-16.

Mgassa MN, Mortensen K, Mbassa GK. The occurrence of bovine lameness in Tanzanian extensive and intensive dairy production systems. XV Congreso Mundial de Buiatria; 11-14 out 1988; Palma de Mallorca, Espanha. León, Espanha: Asociación de Veterinarios Españoles Especialistas en Buiatria; 1988. p.1088-94.

Molina LR, Carvalho AU, Facury Filho EJ, Ferreira PM, Ferreira VCP. Prevalência e classificação das afecções podais em vacas lactantes na bacia leiteira de Belo Horizonte. Arq Bras Med Vet Zootec. 1999;51(2):149-52.

Moraes RR. Caracterização clínica, laboratorial e anatomopatológica da fase inicial da inflamação do tecido interdigital de bovinos da raça Girolanda [dissertação]. Goiânia: Universidade Federal de Goiás; 2000. 110 p.

Nicoletti JLM. Manual de podologia bovina. Barueri: Manole; 2004. 130 p.

Oberbauer AM, Berry SL, Belanger JM, McGoldrick RM, Pinos-Rodriquez JM, Famula TR. Determining the heritable component of dairy cattle foot lesions. J Dairy Sci. 2013;96(1):605-13. 
O’Driscoll K, Gleeson D, O’Brien B, Boyle L. Effect of milking frequency and nutritional level on hoof health, locomotion score and lying behaviour of dairy cows. Livest Sci. 2010;127(2-3):248-56.

Rabelo RE, Vulcani VAS, Sant'Ana FJF, Lima CRO, Rabbers AS, Helrigel PA, et al. Influence of different digital diseases in lameness of dairy cows in southwest of the state of Goiás. R Bras Ci Vet. 2013;20(4):198-203.

Ribeiro PN, Borges JRJ, Ronconi MA, Marsico Filho F, Pitombo CA. Incidência de afecções podais em bovinos de corte abatidos no Estado do Rio de Janeiro. Arq EMVUFBA. 1992;15(1):28-33.

Rowlands GJ, Russell AM, Williams LA. Effects of season, herd size, management system and veterinary practice on the lameness incidence in dairy cattle. Vet Rec. 1983;113(19):441-5.

Schöpke K, Weidling S, Pijl R, Swalve HH. Relationships between hoof disorders, body condition traits, and testday yelds. J Dairy Sci. 2013;96(1):679-89.
Shearer JK, van Amstel SR. Managing lameness for improved cow comfort and performance. Western Management Conference; 12-14 mar 2003; Reno, Nevada. [acesso 20 out 2018]. Disponível em: https://tinyurl. com/yaz7eqa6.

Silva LAF, Silva LM, Romani AF, Rabelo RE, Fioravanti MCS, Souza TM, et al. Características clínicas e epidemiológicos das enfermidades podais em vacas lactantes do município de Orizona - GO. Cienc Anim Bras. 2001;2(2):119-26.

Silveira JAS, Albernaz TT, Oliveira CMC, Duarte MD, Barbosa JD. Afecções podais em vacas da bacia leiteira de Rondon do Pará. Pesq Vet Bras. 2009;29(11):905-9.

Silveira JB, Menechelli AA, Andrade EF, Mathias LA. Levantamento epidemiológico das principais afecções podais em bovinos no município de Votuporanga-SP. Cienc Vet Jabot. 1988;2(2):18-9.

Vermunt JJ, Greenough PR. Predisposing factors of laminitis in cattle. Br Vet J. 1994;150(2):151-64. 